\title{
Iniciativa emprendedora nos viveiros de empresa dos centros de formación profesional
}

\section{Entrepreneurial spirit on the bussiness incubators placed in vocational training schools}

\author{
Serxia Lage Arias* \\ *Dpto. Formación e Orientación Laboral, IES Fernando Wirtz Suárez (A Coruña)
}

\begin{abstract}
Resumo
$O$ fomento da iniciativa emprendedora leva sendo obxecto dun tratamento prioritario no ámbito da educación e formación en Europa nos últimos anos. A competencia emprendedora intégrase no currículo da Formación Profesional (FP) a través da materia "Empresa e Iniciativa Emprendedora" e, nos Centros Integrados de FP, desenvólvese a través de dous dos seus órganos: as coordinacións de emprendemento e os viveiros de empresa. Nesta comunicación dáse conta dos resultados da incipiente acción dos viveiros de empresa dos Centros de Formación Profesional da cidade da Coruña e das súas perspectivas de futuro. A información foi obtida a través dunha entrevista estructurada aplicada a todas as coordinadoras de emprendemento da Coruña.

Palabras clave: Formación Profesional, Orientación Laboral, Viveiros de Empresa, Galicia
\end{abstract}

\begin{abstract}
In the last few years, promoting entrepreneurship has been treated as a priority in European education and training policies. Entrepreneurship Skills are integrated into the Vocational Education and Training (VET) standard curriculum through a subject called "Business and Entrepreneurial Spirit" and, for VET Integrated Schools, these skills have also been developed by two embedded bodies: Entrepreneurship Coordinators and Business Incubators. This communication refers to the results of the newly Business Incubators placed at VET Schools in A Coruña, and also their foreseeable developments. This information was obtained through a structured interview with all the Entrepreneurship Coordinators in A Coruña.

Keywords: Vocational Education, Professional
\end{abstract} Guidance, Business Incubators, Galicia.

\section{Introdución}

A Formación Profesional está a experimentar unha profunda transformación nos últimos anos. A creación dos Centros Integrados de Formación Profesional ven representar un cambio significativo na orientación deste estudos e no xeito de organizar os centros educativos. Así pois, o Decreto 266/2007, do 28 de decembro, polo que se regulan os Centros Integrados de Formación Profesional de Galicia, defíneos como institucións ao servizo da cidadanía e do sector produtivo, que deben contribuír á cualificación e recualificación das persoas, acomodándose ás súas expectativas profesionais.

Tendo en conta que o obxectivo dos centros de FP, e mais en concreto dos CIFP, é atender ás necesidades de cualificación inmediatas e emerxentes, unha saída natural é desenvolver vínculos co sistema produtivo e formativo da súa contorna e informar e orientar ás persoas usuarias, tanto a título individual como colectivamente para que acaden os seus obxectivos persoais e profesionais

O propósito desta comunicación é achegar información sobre os viveiros de empresa situados na cidade da Coruña, concretamente sobre o desenvolvemento práctico do emprendemento nos centros de Formación Profesional.

Para comprender a creación dos viveiros de empresa nos centros educativos, é preciso facer unha contextualización histórico-normativa. $\mathrm{O}$ interese por fomentar a Iniciativa Emprendedora no ámbito educativo e, concretamente, na Formación Profesional xorde dun obxectivo estratéxico da Unión Europea recollido en diversos documentos e declaracións, dende o Consello Europeo de Lisboa celebrado en marzo de 2000 que deseñaba a estratexia Europa 2010 dunha economía baseada no coñecemento, ate a mais recente Estratexia Europa 2020, xurdida da Comisión Europea no 2010 onde traza unha estratexia para un crecemento intelixente, sostible e integrador para Europa.

O Plan de Acción sobre o Emprendemento 2020, publicado pola Comisión Europea no 2013, proponse relanzar o espírito emprendedor en Europa. Neste plan recóllense tres piares para o emprendemento: educar e formar para o emprendemento, crear unha contorna favorable aos emprendedores e establecer modelos e chegar a grupos específicos para emprender. 
Unha das cinco prioridades políticas no ámbito da Educación e Formación en Europa que recolle o programa Erasmus+ é a Iniciativa Empresarial. A competencia emprendedora deberá desenvolverse tanto na adquisición de coñecementos sobre as empresas como no fomento de aptitudes e actitudes esenciais tales como a creatividade, a iniciativa, a tenacidade, o traballo en equipo, o coñecemento do risco e o sentido da responsabilidade.

No ámbito estatal, a Lei Orgánica 5/2002, das cualificacións e da formación profesional, seguindo unha estratexia coordinada para o emprego promovida pola Unión Europea, fíxase como propósito específico a creación dun sistema nacional de Cualificacións e Formación Profesional, cunha ferramenta específica, o Catalogo Nacional de Cualificacións Profesionais, e uns fins específicos entre os que conta: Incorporar á oferta formativa aquelas accións de formación que capaciten para o desempeño de actividades empresariais e por conta propia, así como para o fomento das iniciativas empresariais e do espírito emprendedor que contemplará tódalas formas de constitución e organización das empresas, xa sexan estas individuais ou colectivas, e en especial as da economía social (artigo 3.4)

A Lei Orgánica de Educación 3/2006, de 3 de maio (LOE) establece no seu artigo 40.1 h) que a Formación Profesional no sistema educativo contribuirá a que o alumnado acade os resultados de aprendizaxe que lle permitan afianzar o espírito emprendedor para o desenvolvemento de actividades e iniciativas empresariais.

A entrada en vigor desta LOE, veu sucedida por unha ampla modificación curricular dos Ciclos Formativos de Formación Profesional de Grao Medio e Superior para adaptar as titulacións preexistentes e as de nova implantación aos requirimentos do Sistema de Cualificacións Profesionais, do Catálogo de Cualificacións Profesionais e da mesma LOE. Entre outras novidades, introduciuse un módulo transversal á maior parte das titulacións de FP denominado "Empresa e Iniciativa Emprendedora". Este módulo, de carácter obrigatorio e con atribución docente ao profesorado de Formación e Orientación Laboral, está orientado pedagóxicamente a que o alumnado acade a formación necesaria para desenvolver a propia iniciativa no eido empresarial, tanto cara o autoemprego como cara á asunción de responsabilidades e funcións no emprego por conta allea.

De acordo co disposto nos Reais Decretos que ordenan os títulos de Formación Profesional, neste módulo de Empresa e Iniciativa Emprendedora é preceptivo realizar un proxecto de plan de empresa relacionado coa actividade propia do título e que inclúa todas as facetas de posta en marcha dun negocio, así como a xustificación da súa necesidade social.

$\mathrm{Na}$ normativa autonómica de Galicia o Decreto 114/2010, de 1 de xullo, polo que se establece a ordenación xeral do sistema educativo de Galicia, expón que [a formación profesional] no ámbito do sistema educativo ten por finalidade preparar o alumnado para a actividade nun campo profesional e facilitar a súa adaptación ás modificacións laborais que se poidan producir ao longo da vida, así como contribuír ao seu desenvolvemento persoal, ao exercicio dunha cidadanía democrática e á aprendizaxe permanente. Ademais, na preparación das persoas cobrará singular importancia (...) o espírito emprendedor ( $\operatorname{artigo} 2^{\circ} .2$ )

Así mesmo, no seu artigo $3^{\circ}$ recóllese que as ensinanzas de formación profesional teñen por obxecto conseguir que os alumnos e as alumnas adquiran as capacidades que lles permitan: g) Afianzar o espírito emprendedor para o desempeño de actividades e iniciativas profesionais.

O Decreto 77/2011, do Regulamento Orgánico dos Centros Integrados de Formación Profesional de Galicia, organiza a actividade dos CIFP en xefaturas de áreas e, no que respecta á xefatura da área de calidade, innovación e orientación profesional, establece que nas competencias en materia de información e orientación profesional ten, entre outros cometidos, impulsar proxectos comprometidos coa cultura emprendedora, así como apoiar proxectos empresariais ou de autoemprego e de ideas de negocio que, de ser o caso, poidan xurdir no ámbito do centro integrado, e formular propostas para fomentar a cultura emprendedora nas actividades que se desenvolvan no centro integrado.

Este mesmo Decreto 77/2011, no seu artigo 33 establece as funcións do Coordinador ou Coordinadora de Emprendemento. Nesta enumeración de funcións, queda establecida a existencia dos viveiros de empresa nos centros integrados de FP, a competencia formativa e informativa en emprendemento e a dependencia orgánica e funcional desta coordinación ao Departamento de información e orientación profesional.

A Orde do 29 de xuño de 2011, que desenvolve o Decreto 77/2011 do 7 de abril, recolle no seu artigo 6 que o Plan de Orientación Profesional debe establecer as bases que permitan (...) prestar servizos de atención singularizada ás empresas, nomeadamente a aquelas pequenas e medianas, así como os traballadores autónomos, en relación aos recursos que permitan mellorar o aproveitamento do seu capital humano e deseñar itinerarios formativos axustados ás súas necesidades. No contido mínimo do Plan de Orientación Profesional que se recolle no anexo I da mencionada Orde, é preciso facer referencia específica á estratexia de emprendemento que se desenvolverá no centro.

Para finalizar, a Consellería de Educación da Xunta de Galicia desenvolveu un Plan de Emprendemento no Sistema Educativo de Galicia (Eduemprende), dirixido a todos os centros sostidos con fondos públicos da Comunidade Autónoma de Galicia que impartan os niveis de educación primaria, educación secundaria obrigatoria, bacharelato, programas de cualificación profesional inicial, ensinanzas para persoas adultas, formación profesional, ensinanzas profesionais de artes plásticas e deseño, e ensinanzas deportivas. Porén, as 
accións específicas de fomento do emprendemento no sistema educativo na etapa da Formación Profesional, concrétanse nas accións do programa Eduemprende. Ver figura 1.

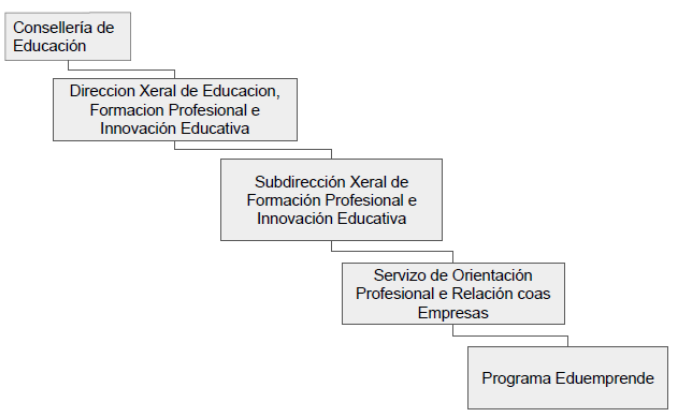

Figura 1. Estrutura institucional do emprendemento educativo nas etapas non universitarias en Galicia

Fonte: elaboración propia baseada en http://www.edu.xunta.es/fp/eduemprende

Para acceder a un viveiro de empresa, as persoas candidatas deberán estar matriculadas, ou ter rematado dentro dos catro anos anteriores á solicitude, algunha ensinanza de carácter post obrigatorio e non universitario, sostida con fondos públicos de Galicia. Neste sentido, os viveiros non só están dirixidos a estudantes ou titulados de Formación Profesional, tamén aos de ensinanzas de réxime especial (artes, música, deportes) e, finalmente, a outras ensinanzas da etapa post-obrigatoria non cualificadas profesionalmente como a Educación Secundaria para Persoas Adultas (ESA) ou Bacharelato.

As persoas candidatas poden integrarse nun proxecto colectivo ou ser asesorados individualmente. Así mesmo poden elixir o centro, dentro da Rede Galega de Viveiros de Empresa, que mellor se axuste ás súas necesidades sen importar que non existira relación académica previa.

No momento da redacción da presente comunicación, a Rede Galega de Viveiros de Empresa está formada por vinte e cinco viveiros, dos cales vinte estaban en funcionamento ao peche do curso 2016-17 e cinco comezarán a funcionar no curso 2017-18.

Para acceder aos servizos do viveiro a persoa candidata deberá completar os seguintes pasos:

1. Entrevista inicial co titor/a do viveiro para valorar a idea emprendedora e o equipo.

2. Elaboración/preparación do plan de empresa, guiado polo titor/a e con dereito a utilizar as instalacións do viveiro de xeito temporal e sen exclusividade, de acordo co plan de traballo acordado co titor/a. Nesta etapa poderán intervir técnicos especialistas externos para verificar a viabilidade económica e legal, así como para asesorar sobre vías de financiamento e posta en marcha do negocio.

3. Unha vez que o proxecto é valorado como viable, a persoa ou persoas emprededoras, de ser un equipo, deberán asinar un contrato tipo de cesión de espazo no viveiro de empresas do centro de FP elixido, para usar de xeito gratuíto as instalacións como sede da empresa durante un máximo de 24 meses.

O coordinador ou coordinadora de emprendemento nos centros de formación profesional forma parte do departamento de información e orientación profesional.

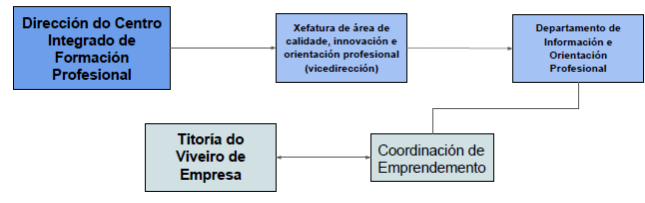

Figura 2: Estrutura do emprendemento dentro dos centros educativos

Fonte: elaboración propia, baseada no Decreto 77/2011.

De acordo co artigo 33, as funcións do coordinador ou coordinadora de emprendemento son:

a) Xestionar os viveiros de empresas e titorizar os proxectos seleccionados con base na súa viabilidade, realizando o seguimento do seu desenvolvemento para facilitar a súa posta en funcionamento.

b) Difundir, promover e apoiar as iniciativas emprendedoras entre todo o alumnado do centro.

c) Coordinar cos centros da súa área de influencia accións de motivación e formación do alumnado que mostre inquedanzas emprendedoras.

d) Informar os posibles emprendedores dos recursos dispoñibles na área de emprendemento, tanto en liña como material físico.

d) Propoñer cursos de formación e actualización do profesorado implicado nesta actividade.

e) Propoñer estadías ou sesións formativas en empresas ou institucións públicas que traballan no sector, en función das necesidades de formación dos participantes na actividade de emprendemento.

f) Crear unha rede de traballo co profesorado do centro e da súa área de influencia, establecendo bancos de ideas que favorezan o desenvolvemento dos sectores do contorno produtivo.

g) Aqueloutras funcións que lle asigne a xefatura do departamento de información e orientación profesional, dentro do seu ámbito de actuación.

\section{Método}

Os resultados que se van presentar foron obtidos a través dunha entrevista estruturada, realizada ás catro coordinadoras dos viveiros de empresa da Coruña, que representan a totalidade dos viveiros na etapa da formación profesional a nivel local e o $20 \%$ dos 
existentes a nivel galego. Tres son titoras de viveiros en funcionamento, cunha traxectoria entre cinco e dous cursos académicos. Unha está a coordinar o próximo lanzamento do viveiro do seu centro no vindeiro curso 2017-18.

As entrevistadas, ademais de titoras ou futuras titoras do viveiro, son tamén as coordinadoras de emprendemento dos seus correspondentes centros, membros do departamento de orientación e información profesional e profesoras de Formación e Orientación Laboral en activo, coa atribución docente do modulo de Empresa e Iniciativa Emprendedora.

O obxectivo primario foi facer unha aproximación ao emprendemento educativo nos centros de formación profesional. Secundariamente pretendíase un achegamento persoal ao profesorado de Formación e Orientación Laboral encargado dos viveiros de empresa para coñecer da súa experiencia, expectativas e necesidades como axentes intermediarios e dinamizadores entre o mundo da formación e do traballo, neste caso enfocado ao autoemprego e a creación de empresas.

As entrevistas desenvolvéronse de xeito presencial durante o mes de xuño de 2017, xeralmente na sede dos propios viveiros, sobre a base dun cuestionario elaborado ao efecto e estruturado en seis dimensións, cun numero de entre dúas e dez preguntas cada unha. A autora é tamén profesora da mesma especialidade, circunstancia que facilitou un ambiente de confianza, digresións e achegas persoais das entrevistadas, que enriqueceron e profundaron nas iniciais dimensións recollidas no cuestionario.

As dimensións sobre as que se estruturaba a entrevista eran as seguintes:

1. Organización, estrutura institucional e responsables do viveiro ( 3 items)

2. Orixe e traxectoria do viveiro (2 items)

3. Actividades desenvolvidas no viveiro (10 items)

4. Principais problemas que se encontran no seu desenvolvemento (4 items)

5. Orzamento e recursos (4 items)

6. Resultados e percepción do éxito (2 items)

Ademais de dar conta das actividades desenvolvidas polos viveiros, coa metodoloxía da entrevista persoal buscouse coñecer a perspectiva e a apreciación particular que sobre o funcionamento dos viveiros e sobre a estratexia de emprendemento desenvolvida pola Administración Educativa teñen as docentes de FP entrevistadas.

\section{Resultados}

A continuación expóñense os resultados, seguindo as dimensións nas que se estruturaba a entrevista.

Os viveiros de empresa dos centros de FP, atendendo ás respostas obtidas ao respecto da organización e estrutura institucional, funcionan como unha extensión da acción orientadora do alumnado e dos usuarios do centro.

No tocante ás actividades desenvolvidas, que se planifican nunha programación didáctica anual, a maior parte das que desenvolven os viveiros están centralizadas pola Consellería de Educación como parte da estratexia de emprendemento ou por outras Administracións como o Concello da Coruña ou a Consellería de Economía a través do IGAPE (Instituto Galego de Promoción Empresarial)

Hai experiencias de integración do viveiro no currículo do modulo de Empresa e Iniciativa Emprendedora (A nosa actividade fundamental $e$ a realización do proxectos de empresa por parte do alumnado. Suxeito 3) Porén, as entrevistadas coinciden en afirmar que a programación de emprendemento ten só carácter básico, con flexibilidade para novas actividades e colaboracións ao longo do curso.

O principal problema co que se encontran os viveiros de empresas nos centros de FP é, curiosamente, a súa localización dentro do centro educativo. Con carácter xeral, as coordinadoras entrevistadas coinciden en sinalar que as limitacións inherentes a esa circunstancia (adaptación ao horario e calendario escolar, falta de privacidade e ausencia de almacén ou taller) son un atranco significativo para transformar un proxecto académico nunha empresa real.

Outras dificultades sinaladas nas entrevistas son a percibida falta de implicación dos departamentos de familia profesional nas accións de emprendemento (suxeitos 1 e 2) a falta de voluntariedade na asunción das tarefas de emprendemento (suxeito 4) e a planificación das actividades centralizadas é pouco acorde co calendario académico (suxeito 1)

Os viveiros de empresa sufráganse con cargo ao orzamento ordinario de funcionamento dos centros educativos. Os recursos que os viveiros poñen a disposición dos usuarios de xeito gratuíto son: equipamento de oficina de uso non exclusivo (mesas, cadeiras, arquivadores, sala de xuntas, equipamento informático), liña de teléfono e rede de internet, servizo de limpeza, mantemento e conserxería.

Dentro das funcións de asesoría, as coordinadoras de emprendemento actúan como titoras do viveiro, dinamizando e orientando aos candidatos en todo o proceso de desenvolvemento da idea de negocio e do proxecto de empresa. Pode contarse tamén coa participación de expertos alleos ao centro educativo para asesorar sobre cuestións específicas da posta en marcha do negocio. As titoras contan con liberación horaria para atender o viveiro, que deben compatibilizar coa labor docente ordinaria. Aínda que na maioría dos casos teñen un horario de atención ao público, é habitual que realicen atención telefónica ou por internet a viveiristas ou candidatos, excedendo o horario regulado.

De xeito informal e non estruturado, os viveiros contan coa asesoría en cuestións técnicas do profesorado de familia profesional.

Ao respecto da traxectoria e resultados acadados, dende o curso 2012-13 os viveiros de empresa da Coruña levan atendidos 77 proxectos de empresa, dos cales 3 chegaron a constituírse legalmente, o que resulta unha taxa de conversión en empresa dun $4 \%$.

Porén, non debemos esquecer que estes viveiros forman parte dun centro educativo e están encadrados 
nunha estratexia de orientación profesional para estudantes e post-graduados. Estes viveiros, na opinión maioritaria das coordinadoras enquisadas, non contan coa estrutura, recursos e localización óptimas para acelerar o desenvolvemento dunha empresa. Daquela, valorar como única medida de éxito a taxa de conversión non semella equitativo.

Así pois, considerando que dende as coordinacións de emprendemento e os viveiros de empresa presentáronse 36 proxectos de empresa a concursos e acadáronse 8 primeiros premios e 7 accésits, a taxa de éxito dende o curso 2011-12 é, daquela, dun $42 \%$.

\section{Conclusións}

A traxectoria dos viveiros de empresa nos centros de formación profesional da Coruña, a pesar da súa brevidade, amósanos un panorama esperanzador aínda que complexo. Tras esta aproximación aos viveiros da locais, xorde a cuestión de si estes resultados serán semellantes na provincia ou no resto de Galicia. Así mesmo, cómpre contar coa visión e experiencia dos viveiristas e usuarios dos servizos do viveiro.

Así pois, tendo en conta os principais problemas detectados, sería conveniente considerar unha localización dos viveiros dentro dos recintos académicos pero coa independencia e autonomía necesarias para desenvolver actividades profesionais. É tamén preciso reforzar o traballo en rede e o contacto entre as coordinacións de emprendemento, así como implementar canles mais dinámicas de contacto co tecido empresarial local, fomentando a mentoría e o estudio de casos de éxito.

Os viveiros de empresa nos centros de FP, daquela, deben ser considerados mais aló dunha función de aceleradores empresariais, se non como unha contribución á cultura do emprendemento educativo, entendida como aquela baseada na creatividade, na tenacidade, na asunción do risco, no traballo en equipo e no desenvolvemento de iniciativas tanto individuais como colectivas que teñan un compoñente transformador da contorna, en especial aquelas orientadas á economía social.

\section{Referencias}

Diz López, M.J. (2013). Los centros integrados de formación profesional de Galicia. Desarrollo normativo e implantación. Innovación educativa, 23, pp. 255-258. http://www.usc.es/revistas/index.php/ie/article/view/1 330/1514

Ferreiro Seoane, F.J. (2013) Los viveros de empresa de Galicia. Una estrategia generadora de riqueza. (Tese de doutoramento) Facultade de Economía e Empresa. Departamento de Economía Aplicada I. Universidade da Coruña. Recuperada de http://hdl.handle.net/2183/11807

Rego, L., Barreira, E., \& Mariño, R. (2015). Apertura dos centros de formación profesional á contorna local: percepción dos axentes sociais. Revista de
Estudios e Investigación en Psicología y Educación, 0(07), 023-027. doi:http://dx.doi.org/10.17979/reipe.2015.0.07.351

Sobrado Fernández, L., Fernández Rey, E. (2010) Competencias emprendedoras y desarrollo del espíritu empresarial en los centros educativos. Educación XXI. 13(1), pp. 15-38. doi: http://dx.doi.org/10.5944/educxx1.13.1.275

Consello Europeo de Lisboa, celebrado o 23 e 24 de marzo de 2000. Conclusións da Presidencia. http://www.europarl.europa.eu/summits/lis1_es.htm

Comisión Europea: Plan de Acción sobre Emprendemento 2020. COM (2012) 795 final

Erasmus+ Programme Guide. Version 2 (2017): 20/01/2017

http://sepie.es/doc/convocatoria/2017/erasmus-plusprogramme-guide env2.pdf

Lei Orgánica 5/2002, das cualificacións e da formación profesional.

Lei Orgánica 1/2006, de Educación.

Decreto 266/2007, dos centros integrados de formación profesional de Galicia.

Decreto $114 / 2010$, de ordenación xeral do sistema educativo de Galicia.

Decreto 77/2011, Regulamento orgánico dos centros integrados de formación profesional de Galicia

Plan de Emprendemento do Sistema Educativo de Galicia-Eduemprende http://www.edu.xunta.es/fp/webfm_send/1930 